\title{
BMJ Open Cancer Aging Research Group (CARG) score in older adults undergoing curative intent chemotherapy: a prospective cohort study
}

\author{
Vikas Ostwal (D , , Anant Ramaswamy, ${ }^{1}$ Prabhat Bhargava, ${ }^{1}$ \\ Tejaswee Hatkhambkar, ${ }^{1}$ Rohit Swami, ${ }^{2}$ Sameer Rastogi, ${ }^{3}$ Sarika Mandavkar, ${ }^{1}$ \\ Jaya Ghosh, ${ }^{1}$ Jyoti Bajpai, ${ }^{1}$ Seema Gulia, ${ }^{1}$ Sujay Srinivas, ${ }^{1}$ Sushmita Rath, ${ }^{1}$ \\ Sudeep Gupta (iD ${ }^{1}$
}

To cite: Ostwal V,

Ramaswamy A, Bhargava P, et al. Cancer Aging Research Group (CARG) score in older adults undergoing curative intent chemotherapy: a prospective cohort study. BMJ Open 2021;11:e047376. doi:10.1136/ bmjopen-2020-047376

- Prepublication history and additional supplemental material for this paper are available online. To view these files, please visit the journal online. To view these files, please visit the journal online (http://dx.doi. org/10.1136/bmjopen-2020 047376).

Received 30 November 2020 Accepted 08 June 2021

D) Check for updates

(c) Author(s) (or their employer(s)) 2021. Re-use permitted under CC BY-NC. No commercial re-use. See rights and permissions. Published by BMJ.

${ }^{1}$ Department of Medical Oncology, Tata Memorial Centre, Homi Bhabha National Institute, Mumbai, Maharashtra, India

${ }^{2}$ Department of Medical Oncology, Narayana Multispeciality Hospital, Jaipur, Rajasthan, India

${ }^{3}$ Department of Medical Oncology, AlIMS, New Delhi, India

Correspondence to Dr Anant Ramaswamy; anantr13@gmail.com

\section{ABSTRACT}

Importance The Cancer Aging Research Group (CARG) toxicity score is used to assess toxicity risk in geriatric patients receiving chemotherapy.

Objective The primary aim was to validate the CARG score in geriatric patients treated with curative intent chemotherapy in predicting grade 3-5 toxicities.

Design This was a longitudinal prospective observational study.

Setting Tata Memorial Hospital, Mumbai, India, a tertiary cancer care referral centre.

Participants Patients, aged $\geq 65$ years, with gastrointestinal, breast or gynaecological stage I-III cancers being planned for curative intent chemotherapy. A total of 270 patients were required for accrual in the study. Exposure(s) Total risk score ranged from 0 (lowest toxicity risk) to 19 (highest toxicity risk).

Main outcome(s) and measure(s) The primary endpoint of the study was to evaluate whether the CARG risk score predicted for grade 3-5 toxicities.

Results The study cohort of 270 patients had a mean age of 69 (65-83) years, with the most common cancers being gastrointestinal $(79 \%)$. Fifty-two per cent of patients had atleast one grade $3-5$ toxicity. The risk of toxicity was increased with an increasing risk score (42\% low risk, $51 \%$ medium risk and $79 \%$ high risk; $p<0.001)$. There was no association between either Eastern Cooperative Oncology Group (ECOG) performance status $(\mathrm{p}=0.69)$ or age-adjusted Charlson Comorbidity Index ( $p=0.79$ ) risk categories and grade $3-5$ chemotherapy toxicities.

Conclusions and relevance This study validates the CARG risk score in predicting for grade 3-5 toxicities in geriatric oncology patients receiving curative intent chemotherapy and can be considered as the standard of care before planning chemotherapy in every elderly patient.

Trial registration number CTRI/2016/10/007357; Results.

\section{INTRODUCTION}

Older adult patients (age $\geq 65$ years) with cancer represent a growing proportion of patients in community clinical practice,
Strengths and limitations of this study

- The Cancer Aging Research Group (CARG) risk score is a simple tool comprising easily available clinical information.

- This is a prospective study to assess CARG risk score in elderly patients treated with curative intent chemotherapy to predict for grade 3-5 toxicities.

- CARG score performed better than traditional indices such as the age-adjusted Charlson Comorbidity Index and ECOG performance status.

- The results suggest that the CARG score is valid in the studied population and can be routinely used in clinical practice.

- This study does not include palliative patients and mainly patients with gastrointestinal cancers were recruited.

primarily due to increasing life-spans as well as medical progress contributing to decreased morbidity and mortality from other causes. ${ }^{1}$ Elderly patients comprise anywhere between $20 \%$ and $60 \%$ in community oncology practice, with variances based on access to cancer care, disease stage and centre-specific management strategies. ${ }^{2} 3$

The age-adjusted Charlson Comorbidity Index (ACCI) and ECOG performance status (PS) among others have often been used to quantify risks and predict for outcomes in older adults with cancer, but there are limited data for correlation between these indices and treatment-related side effects. ${ }^{4-6}$ The Cancer Aging Research Group (CARG) risk score, developed by Hurria and colleagues, ${ }^{78}$ is an easy-to-use tool that predicts for significant chemotherapy-related toxicities (grades 3-5) in older North American adults aged $\geq 65$ years starting on chemotherapy. Based on their training samples and subsequent 
validation studies, the investigators clearly identified low-risk, mid-risk and high-risk groups predicting for increasing rates of grade 3-5 toxicities (low-risk: $30 \%$, intermediate-risk: $52 \%$ and high-risk: $83 \%$ ) with statistical significance $(\mathrm{p}<0.001)$. The CARG risk score has been validated in other countries and in specific tumour sites to varying degrees. ${ }^{9} 10$

In older adults being treated with curative intent chemotherapy, there is the possibility of treating oncologists using standard doses to maximise outcomes, despite patient-related indicators suggesting a requirement for lower doses. This is a unique scenario where further information on risks and benefits would allow for informed clinical decision-making on doses and drugs to be used. As patients with all stages of cancer were included in the CARG studies, the ambiguity with regard to its usage in patients being treated with potentially curative intent chemotherapy lends itself to re-examination.

With this background, the investigators conducted a longitudinal prospective study with the primary aim of validating the CARG risk score in older Indian patients receiving curative intent chemotherapy (neoadjuvant or/and adjuvant chemotherapy) for cancer. Secondary objectives included correlation of the ACCI and physician measured ECOG PS with grade 3-5 toxicities. An exploratory component of the study involved an estimation of grade 1 and grade 2 toxicities and their correlations with the CARG risk score.

\section{MATERIALS AND METHODS \\ Patient selection and design}

The study was designed as a longitudinal prospective observational study to validate the CARG risk score in predicting chemotherapy toxicity risk in elderly patients. The study was conducted at the Tata Memorial Hospital and enrolled consecutive patients aged $\geq 65$ years, chemotherapy naive, with a histological diagnosis of gastrointestinal, breast or gynaecological cancer, stage I-III diseases and planned for neoadjuvant or adjuvant systemic chemotherapy as a potentially curative treatment option.

The study was designed by investigators from the Department of Medical Oncology of the Tata Memorial Hospital.Written informed consent was obtained from all patients before inclusion in the study.

\section{Patient and public involvement}

There was no public or patient involvement in design, conduct or results declaration of the study.

\section{Study procedures}

Data regarding tumour type and stage, pretreatment laboratory values and chemotherapy regimen were recorded. All patients underwent standard prechemotherapy workup, including evaluation of end-organ function. Patients were planned for chemotherapy by treating oncologist (with an assessment of ECOG PS and ACCI), who was blinded to the risk score. A trained medical doctor calculated the CARG risk score for patients enrolled in the study. The assessment of the score by the trained medical doctor was independently reviewed by an oncologist who was not part of the treating team. ${ }^{7}$ Total risk score ranged from 0 (lowest toxicity risk) to 19 (highest toxicity risk), with division of the scores into low risk ( $0-5$ points), intermediate risk (6-9 points) and high risk (10-19 points) as per the classification in the original study by Hurria $e t \mathrm{al}^{7}$ One modification of the original CARG risk score which was used in this study was the measurement of 'Walking 1 block'. The concept of measuring distances by a block is not prevalent in India and hence, a distance of $100 \mathrm{~m}$ in the immediate vicinity of the hospital was measured and patients were scored on their ability to walk the same. The chemotherapy dosing for the first cycle of chemotherapy was categorised as 'standard' if $100 \%$ doses were planned and 'dose reduced' if any dose $<100 \%$ was used. The decision for dose modifications, whether initial or subsequent, was based on assessment by treating oncologist. Besides CARG risk score, the ACCI was calculated for all patients as part of standard assessment of older adults with cancer. A cut-off of 4 points $(\leq 4$ and $>4)$ was used to differentiate between low and high CCI scores. ${ }^{11}$ Patients were followed from beginning to the end of chemotherapy course across all cycles of therapy, though occurrence of a single-grade 3-4 toxicity was considered as an endpoint for the purpose of toxicity calculation in the study. Toxicities were captured prospectively at all clinical visits (by treating oncologist and trained medical doctor) and graded as per National Cancer Institute Common Terminology Criteria for Adverse Events, version 4.0. Decision on relatedness of toxicity to chemotherapy was made by treating physician. Laboratory values were captured as grade 1-5 toxicities if they met the criteria on the date of scheduled chemotherapy or when patient was seeking attention because of treatment-related toxicities.

\section{Outcomes}

The primary outcome of the study was the occurrence of any grade 3-5 chemotherapy-related toxicities over the course of planned treatment and its association with the CARG risk score. The planned secondary endpoints of the study were the correlation of ACCI and ECOG PS with grade 3-5 chemotherapy-related toxicities. Occurrence of any grade 1 and 2 chemotherapy-related toxicities and its correlation with CARG risk score was an exploratory aspect of the study.

\section{Statistical analysis}

Descriptive analyses were performed to enumerate patient, tumour, treatment characteristics, CARG risk scores and ACCI. The incidence of grade 3-5 toxicities was calculated and compared between CARG risk groups and ECOG PS cohorts using the $\chi^{2}$ test. The CARG risk score is not routinely used in clinical practice in our institution and we did not have baseline data for the same for the purpose of sample size calculation . We conducted an internal audit of elderly patients with breast, 
gastrointestinal and gynaecological cancers receiving curative intent chemotherapy in our hospital and found a $20 \%$ incidence of grade $3-5$ toxicities in elderly patients with ECOG PS $0 / 1$ and controlled or absent comorbidities (surrogate for 'low risk') as opposed to $36 \%$ in elderly patients with ECOG PS 2 with or without multiple uncontrolled comorbidities (surrogate for 'high risk'). Extrapolating these results, a power of $80 \%$ and $\alpha$ of $5 \%$ with one-sided assumption was required with an estimated sample size required being 246 patients. Assuming an attrition rate of $10 \%$, a total of 270 patients were required for enrolment in the study. The $\chi^{2}$ test was performed to test the association of the CARG risk score, PS and ACCI with grade 3-5 toxicities and for association of CARG risk score with grade 1 and 2 toxicities as well dose modifications. The predictive ability of the CARG risk score was evaluated by calculating receiver-operating characteristic (ROC) curves and calculating the area under the curve (also known as C-statistic). ROC curves were also calculated for ECOG PS and ACCI. All analyses were performed using SPSS V.25. All tests were two-sided, and a $\mathrm{p}$ value of $<0.05$ was considered statistically significant.

\section{RESULTS}

\section{Patient and treatment characteristics}

The study completed accrual of 270 patients, with mean age of patients being 69 (range: 65-83) years, 121 (45\%) female patients and 212 patients $(79 \%)$ having gastrointestinal cancers. For purposes of comparison, data from the seminal CARG study by Hurria $e t$ al are provided for comparison (table 1). Details of chemotherapeutic regimens are presented in online supplemental table 1.

\section{Chemotherapy toxicity}

At least one grade $3-5$ toxicity was seen in 140 patients (52\%), with 119 (44\%) having grade 3, 22 (8\%) having grade 4 and 11 (4\%) grade 5 toxicities. Grade 3-5 haematological and non-haematological toxicities occurred in 60 patients (22\%) and $120(45 \%)$ patients, respectively. Common haematological toxicities were neutropenia in $26(10 \%)$ and febrile neutropenia in $17(6 \%)$ patients, while common non-haematological toxicities were infections, fatigue and diarrhoea in 54 (20\%), 24 (9\%) and 23 (9\%) patients respectively (table 2 ).

The incidence of grade 1 to grade 2 toxicities is listed in online supplemental table 2.

\section{Correlation of CARG score with toxicity and dose modifications}

The median overall CARG risk score was 6 (range, 0-19). Of the 270 patients, $72(27 \%), 164(61 \%)$ and $34(13 \%)$ were classified as low-risk, intermediate-risk and highrisk, respectively (table 1$)$. Grade $3-5$ toxicities were seen in $30(42 \%), 83(51 \%)$ and $27(79 \%)$ patients with lowrisk, intermediate-risk and high-risk score. There was a significant difference in toxicity among the risk groups ( $<<0.001$; figure 1 and table 2$)$. The odds of a patient classified as intermediate risk having a grade $3-5$ toxicity as compared with patient with low risk was 1.64 (95\% CI: 1.23 to 2.13), while the odds of a patient classified as high risk having a grade $3-5$ toxicity as compared with patient with low risk was 7.58 (95\% CI:2.61 to 21.73). Area under the ROC curve for the predictive model in this cohort was 0.63 (95\% CI: 0.57 to 0.7 ). The correlation of individual components of the CARG risk score with grade 3-5 toxicities is enumerated in online supplemental table 3 .

Grade 1 and 2 toxicities were seen in 61 (86\%), 144 $(88 \%)$ and $29(85 \%)$ patients with low-risk, intermediaterisk and high-risk score. There was no significant difference in toxicity among the CARG risk groups $(\mathrm{p}=0.79)$.

The incidences of grade 2 peripheral neuropathy and grade 2 hand-foot-syndrome (HFS) are separately reported as these are specifically associated with diminished function. The incidence of grade 2 neuropathy was seen in $5(7 \%)$, $11(7 \%)$ and $2(6 \%)$ patients in the low-risk, intermediaterisk and high-risk categories, respectively. There was no significant difference in grade 2 neuropathy among the CARG risk groups $(\mathrm{p}=0.97)$. The incidence of grade 2 HFS was seen in $5(7 \%), 18(11 \%)$ and $2(6 \%)$ patients in the low-risk, intermediate-risk and high-risk categories, respectively. There was no significant difference in grade 2 HFS among the CARG risk groups $(\mathrm{p}=0.47)$.

Upfront dose modifications in chemotherapy regimens were performed in 65 patients $(24 \%)$. Subsequent dose reductions were made in 89 patients $(33 \%)$. On further analysis, these subsequent dose modifications were made in 18 (25\%), 59 (36\%) and 12 (35\%) patients in the low-risk, intermediate-risk and high-risk categories, respectively. The differences in proportion of dose modifications were not statistically significant between the three groups $(\mathrm{p}=0.244)$.

\section{Association of grade $\mathbf{3} \mathbf{- 5}$ toxicities with ACCI and ECOG PS}

The median ACCI was 5. A Charlson Comorbidity Index (CCI) $\leq 4$ was seen in 111 patients $(41 \%)$, while 159 patients $(59 \%)$ had a CCI $\geq 4$. There was no significant difference in toxicities among both groups of patients ( $\mathrm{p}=0.7$; figure 1 and table 3 ). The ROC of the model with CCI (as a continuous variable) was 0.48 (95\% CI: 0.41 to 0.55 ), which was lower than the ROC of the CARG risk score model, 0.63 .

ECOG PS was 0, 1 and 2 in 9 (3\%), $221(82 \%)$ and $40(15 \%)$ patients, respectively. There was no significant difference in toxicities among both groups of patients ( $p=0.69$; figure 1 and table 3 ). The ROC of the model with ECOG PS (as a continuous variable) was $0.52(95 \%$ CI: 0.45 to 0.59 ), which was lower than the ROC of the CARG risk score model, 0.63.

\section{DISCUSSION}

This study validates the CARG risk score in older Indian patients receiving curative intent chemotherapy for stages I-III gastrointestinal, breast and gynaecological cancers, though the association between rates of severe chemotherapy toxicity and CARG risk groups as being 
Open access

Table 1 Baseline characteristics of patients

\section{Characteristic}

Mean age in years (range)

Gender

\section{Female}

Male

Comorbidities

Hypertension

Diabetes mellitus

Coronary artery disease

Chronic kidney disease

Number of comorbidities

\begin{tabular}{|c|c|c|}
\hline 0 & $125(46)$ & $10 \%$ \\
\hline 1 & $95(35)$ & - \\
\hline$\geq 2$ & $50(19)$ & - \\
\hline \multicolumn{3}{|l|}{ Cancer stage } \\
\hline Stages I-III & 270 (100) & 191 (38) \\
\hline Undergone resection & $210(78)$ & - \\
\hline \multicolumn{3}{|l|}{ ECOG performance status (clinician assessed) } \\
\hline $0 / 1$ & $230(85)$ & $402(80)$ * \\
\hline 2 & $40(15)$ & $86(17) \dagger$ \\
\hline \multicolumn{3}{|l|}{ Factors assessed in CARG } \\
\hline Age $\geq 72$ years & $60(22)$ & $270(54)$ \\
\hline Cancer type Gl or Genitourinary & $212(79)$ & $185(37)$ \\
\hline Chemotherapy dosing, standard dose & $205(76)$ & $380(76)$ \\
\hline No. of chemotherapy drugs, polychemotherapy & $194(72)$ & $351(70)$ \\
\hline Haemoglobin $<110 \mathrm{~g} / \mathrm{L}$ (male), $<100 \mathrm{~g} / \mathrm{L}$ (female) & $99(37)$ & $62(12)$ \\
\hline Creatinine clearance $<34 \mathrm{~mL} / \mathrm{min}$ & $5(2)$ & $44(9)$ \\
\hline Hearing, fair or worse & $19(7)$ & $123(25)$ \\
\hline No. of falls in last 6 months, 1 or more & $18(7)$ & $91(18)$ \\
\hline IADL: Taking medications, with some help/unable & $29(11)$ & $39(8)$ \\
\hline MOS: Walking 1 block, somewhat limited/limited a lot & $17(6)$ & $109(22)$ \\
\hline $\begin{array}{l}\text { MOS: Decreased social activity because of physical/emotional health, limited at } \\
\text { least sometimes }\end{array}$ & $18(7)$ & $218(44)$ \\
\hline Median overall risk score & 6 & 7 \\
\hline \multicolumn{3}{|l|}{ Risk stratification } \\
\hline Low risk (0-5 points) & $72(27)$ & $128(26)$ \\
\hline Intermediate risk (6-9 points) & $164(61)$ & $227(45)$ \\
\hline High risk (10-19 points) & $34(13)$ & $109(22)$ \\
\hline \multicolumn{3}{|l|}{ Age-adjusted Charlson's Comorbidity Index } \\
\hline$\leq 4$ & $111(41)$ & - \\
\hline$>4$ & 159 (59) & - \\
\hline
\end{tabular}

${ }^{*}$ Equivalent to KPS $\geq 80$.

†Equivalent to KPS 60-70.

CARG, Cancer Aging Research Group; Gl, gastrointestinal; IADL, Instrumental activities of daily living; KPS, Karanofsky Performance Status. cohort $(n=500)$

number $(\% ; n=270)$

$73(65-91)$

$281(56)$

$149(55)$

$219(44)$

$114(42) \quad 52 \%$

$71(26)$

$12(4)$

$-$

$20 \%$

$3(1)$

$-$

CARG training 
Table 2 Treatment-related grade 3-5 toxicities

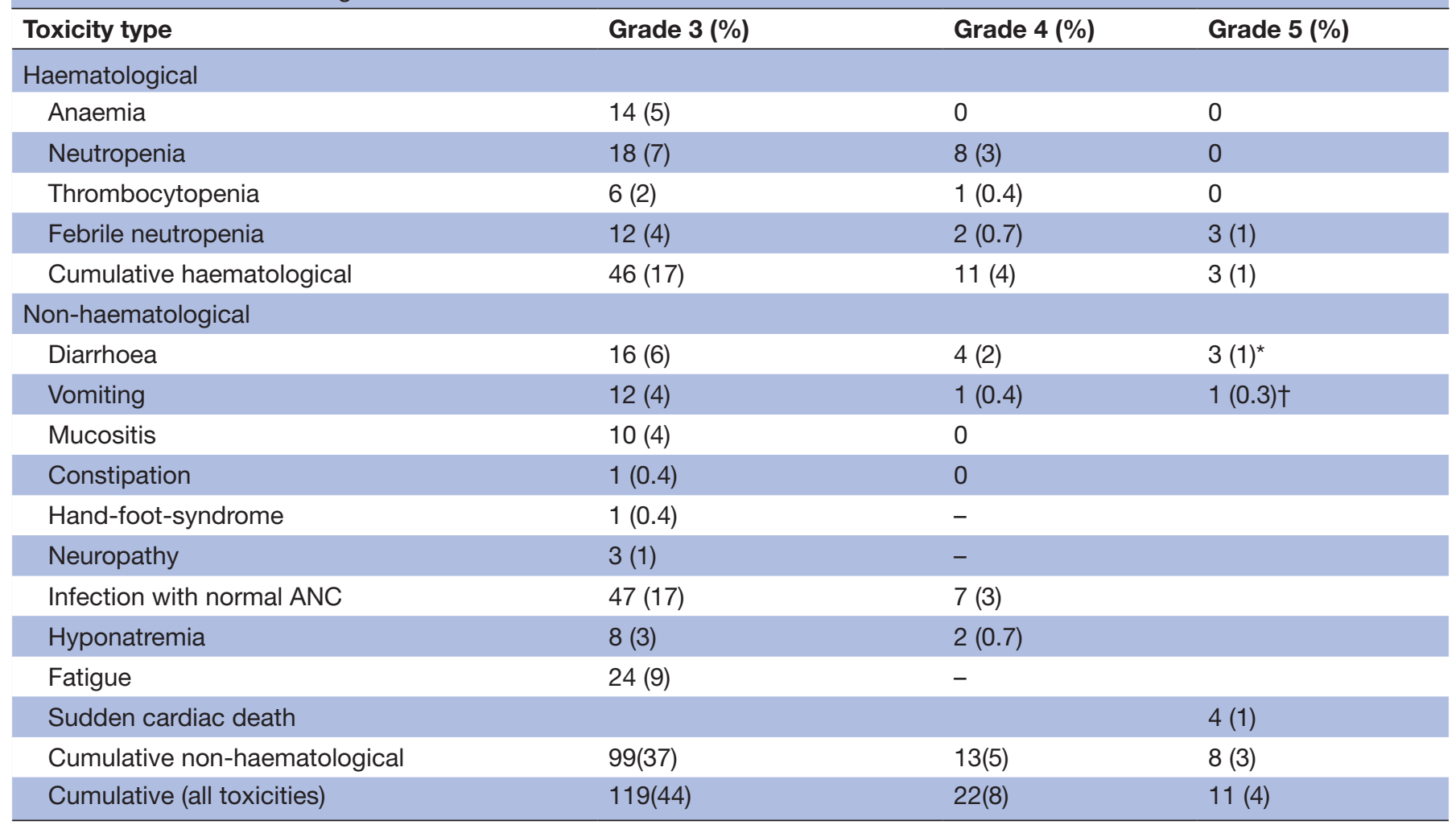

${ }^{*}$ All three patients developed dehydration with resulting acute renal failure.

†Patient developed grade 4 vomiting with irreversible grade 4 hyponatremia resulting in death.

ANC, Absolute neutrophil count.

discriminatory was modest (area under the ROC, 0.63). No association was found between ECOG PS and burden of comorbidities as measured by the ACCI with severe chemotherapy-related toxicities.

There is a significant knowledge gap in terms of how older patients in general and older patients with cancer fare in the Indian scenario. Limited data suggest no defined care structure for older patients with cancer in India as well as only low-moderate awareness and use of geriatric assessment in older patients with cancer. ${ }^{3} 12$ Available evidence from India suggests that $98 \%$ of older adult patients with cancer have vulnerabilities in at least one geriatric domain, though the specific vulnerabilities appear to differ from the previously published data. ${ }^{13}$ Such a high and differential vulnerability profile in these patients suggests that they may have a different incidence of toxicities with standard chemotherapy regimens. With such a background, it was essential to evaluate the validity of the CARG risk score before routine advocation in older adult patients.

There are some important differences between the populations of this study and the seminal CARG study. This study had only patients with stages I-III disease, while the CARG study had $38 \%$ with non-metastatic disease. Other relevant differences between the cohorts include a younger mean age (69 vs 73 years), lesser comorbidities $(46 \%$ with no comorbidities vs $10 \%$ with no comorbidities) and better performance on a number of individual variables in the CARG risk score (better hearing, lesser number of falls, better social activity and effort tolerance). There were also a lower proportion of patients with high-risk score in the current study $(13 \%$ vs $22 \%$ ). These differences, coupled with lack of patients with metastatic disease in the study cohort, indicate that patients in this study were a well preserved and presumably fitter group of patients with lesser disease burden and potential for toxicities.

Despite the differences in patient cohorts in terms of baseline characteristics, this study validated the CARG risk score in predicting grade $3-5$ chemotherapy-related toxicities. The low-risk, intermediate-risk and high-risk CARG groups predicted for increasing incidences of grade 3-5 toxicities with statistical significance. The ORs between individual risk groups for predicting grade 3-5 toxicities was also statistically significant, highlighting the differential capability of the risk score. An unanswered component of the CARG risk assessment was whether it correlated with grade 1 and 2 toxicities. Previous studies by Moth $e t a l^{14}$ estimating grade 1 and grade 2 toxicities as toxicity burden have not shown a correlation with the CARG risk score. This is possibly due to the near universal occurrence of such toxicities in patients receiving chemotherapy. A similar trend was seen in this study wherein an increasing risk score did not predict for an increased risk 


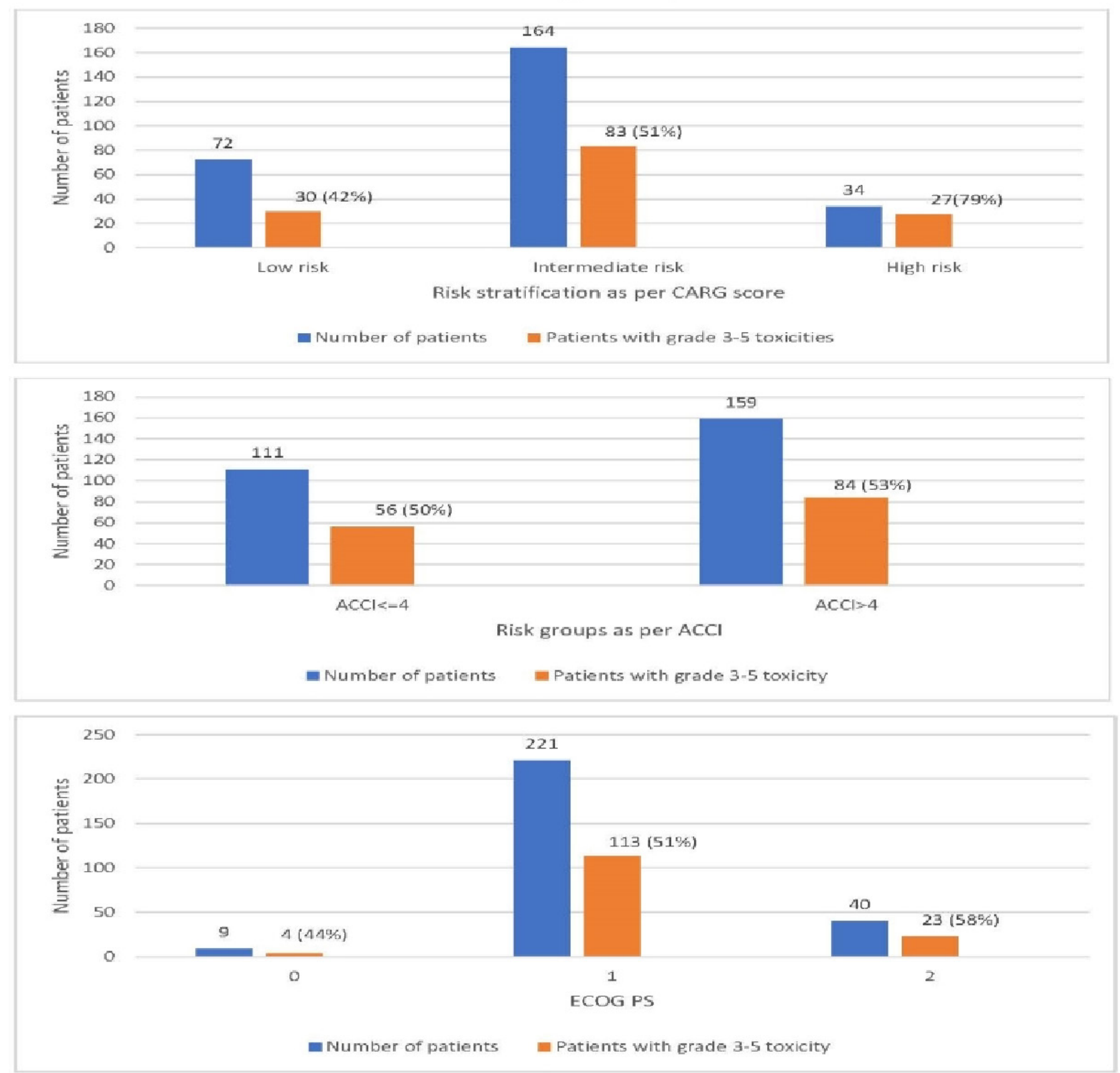

Figure 1 CARG (A) versus (B) ACCl versus (C) ECOG PS predict grade 3-5 toxicities. ACCl, age-adjusted Charlson Comorbidity Index; CARG, Cancer Aging Research Group; PS, performance status.

of grade 1 and 2 toxicities. Additionally, in comparison to the predictive capacity of the CARG risk score, the CCIbased and ECOG PS-based risk groups did not predict for incidences of toxicity in the study. These results highlight certain salient points in the study. First, the CARG risk score can be used with confidence in the Indian population to predict for grade $3-5$ toxicities. The CARG risk score was evaluated only in a North American elderly adult cohort initially and this study provides validation for the score in the Indian context. Second, despite being a betterpreserved cohort in comparison to the population in the seminal study as well as having only patients on curative intent therapy, a high proportion of patients across risk groups developed grade 3-5 toxicities which may be lifethreatening. Thus, it is imperative to carefully assess the trade-off between objectives such as survival and downstaging versus potentially life-threatening toxicities while planning curative intent chemotherapy in older adult patients. Third, the area under the ROC for this study was 0.63 and is lower in comparison to the original study (0.72), though very similar to the results of the validation study (0.65) by the CARG group. ${ }^{8}$ Although this indicates a modest discriminatory capability for the CARG risk score in this study, it is probably also reflective of the true value of the score in prediction of severe chemotherapy-related toxicities. Smaller studies by Australian investigators have 
Table 3 Ability of CARG risk score versus physician-assessed ECOG PS versus ACCI to predict grade 3-5 chemotherapy toxicities

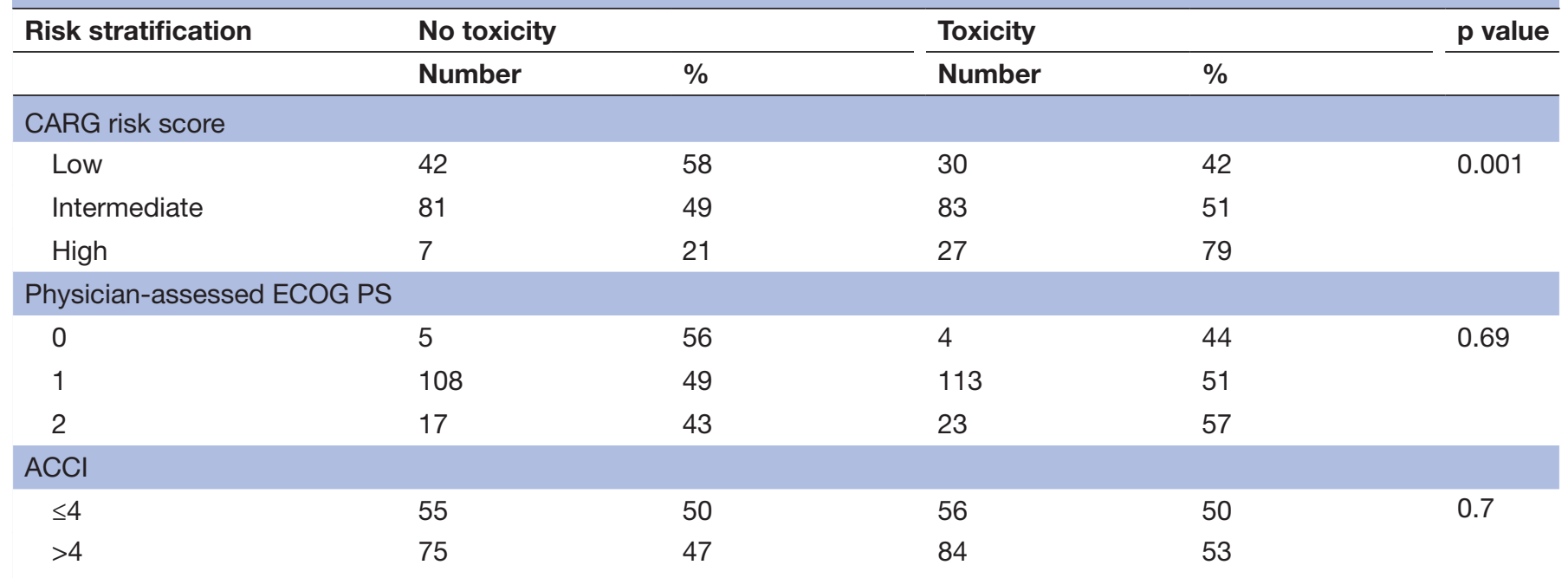

ACCI, age-adjusted Charlson Comorbidity Index; CARG, Cancer Aging Research Group; PS, performance status.

also previously commented on this lack of discriminatory value with the CARG risk score. ${ }^{14}$ Finally, using a global assessment score such as ECOG PS or only one aspect of an assessment profile such as comorbidity status (as in the case of ACCI) would not accurately capture the heterogeneity of the older adult population. This is reflected in the inadequacy of ECOG PS and ACCI in predicting for toxicities and hence, these indices should only be used in conjunction with other indices as measures of assessment in older adults with cancer. ${ }^{1516}$

We also attempted to correlate the CARG risk scores with the necessity for further dose reductions during chemotherapy. There were no statistically significant differences between the risk groups in terms of requirement for subsequent dose modifications post initiation of therapy. This can partially be explained by the fact that a high proportion of patients $(24 \%)$ underwent initial dose reductions when planned for therapy by the treating physicians who were blinded to the CARG risk score. Such an upfront dose reduction may have masked any possible correlation between the risk scores and need for dose modifications during chemotherapy.

Certain strengths of this study need to be highlighted. The prospective collection of toxicity data removes any recall bias that may lead to underestimation of the same. The assessment in patients undergoing curative intent treatment only is novel and lays stress on the conundrum faced by oncologists when balancing risks and benefits of using potentially aggressive chemotherapy regimens in the neoadjuvant or adjuvant setting. The results will allow patients and oncologists to discuss options with evidentiary basis for expected toxicities when treatment regimens are considered. By validating the CARG risk score in an Indian population, the study provides further evidence for the use of the score across geographical regions.
There are certain limitations to this study. This is a singlecentre study and the results may not be generalisable to practice across India. There is an under-representation of non-gastrointestinal cancers and this may hamper the generalisation of the study results to all solid tumours. Additionally, other common solid tumours like lung cancers, head and neck cancers and genitourinary cancers have not been evaluated in this study. The rate of grade 3-5 toxicities was much higher than planned as per baseline statistical considerations - this may relate to the preponderance of gastrointestinal cancers in the study population, besides other differences in baseline characteristics of the patient cohorts as has been previously discussed. Additionally, while information with regard to correlation of the CARG risk score with grade 1 and 2 toxicities has been provided, the relevance of this is limited due to the fact that almost all patients on systemic therapy develop some grade 1 or 2 toxicity. Again, the CARG score was developed to predict for grade 3-5 toxicities, not grade 1 and 2 toxicities and thus, the inability to differentially predict for grades 1 and 2 in this study is not surprising. We also do not have information on patient-related outcomes in the study.

Going forward, future directions with regard to the CARG risk assessment include developing paradigms for the degree of dose modifications required in patients based on the score. Patients preferences with regard to tumour-related endpoints versus toxicity-limiting Quality of life (QOL) based on toxicity risk assessment can be explored in trials, especially in the advanced cancer setting. Non-chemotherapeutic systemic treatment options like targeted therapy and immunotherapy can be assessed by the risk score for predicting toxicity. Based on this study, we plan to use the CARG score routinely in our hospital as well plan prospective studies using the score to estimate dose modifications in relation to risk assessment by the score. 
In conclusion, this study validates the CARG risk score in predicting for grade $3-5$ toxicities in Indian older adult patients with cancer receiving curative intent chemotherapy. The score contributes to informed clinical decision making with regard to planning treatment and expectation of toxicity in this cohort of patients. Additionally, indices such as ECOG PS and CCI are inadequate to predict for toxicities and should only be used along with other measures to predict for chemotherapyrelated toxicities.

Contributors V0 and AR: conception and design, analysis and interpretation of data, drafting of manuscript, final approval of the submitted version. PB, RS, SM, JG, SR, JB, SG and SS: analysis and interpretation of data, drafting of manuscript, final approval of the submitted version. TH: analysis and interpretation of data, final approval of the submitted version. SR: conception and design, drafting of manuscript, final approval of the submitted version.

Funding Received grants to Tata Memorial Centre, Mumbai for the conduct of this study from (a) Indian Association of Supportive Care in Cancer awarded research award during BEST OF MASCC 2019, grant number: 1716-A. (b) Intas Pharmaceuticals Private Limited, grant number: 1716-B. The Pharmaceutical company, Intas Pharmaceutical Private Limited, provided an unrestricted educational grant to Tata Memorial Centre (The Institute) for the conduct of the study. This was used for appointing a trained medical doctor for the purpose of the study.

Competing interests None declared.

Patient consent for publication Not required.

Ethics approval Name of the ethics committee: Institutional Ethics Committee, Tata Memorial Centre, Parel, Mumbai. IEC approval ID: IEC/1019/1716/001. The participants gave informed consent before taking part in the study.

Provenance and peer review Not commissioned; externally peer reviewed.

Data availability statement Data are available upon reasonable request. Deidentified data will be made available after approvals from respective institutional Ethics Committees and proper in place Memorandum of Understanding.

Supplemental material This content has been supplied by the author(s). It has not been vetted by BMJ Publishing Group Limited (BMJ) and may not have been peer-reviewed. Any opinions or recommendations discussed are solely those of the author(s) and are not endorsed by BMJ. BMJ disclaims all liability and responsibility arising from any reliance placed on the content. Where the content includes any translated material, BMJ does not warrant the accuracy and reliability of the translations (including but not limited to local regulations, clinical guidelines, terminology, drug names and drug dosages), and is not responsible for any error and/or omissions arising from translation and adaptation or otherwise.

Open access This is an open access article distributed in accordance with the Creative Commons Attribution Non Commercial (CC BY-NC 4.0) license, which permits others to distribute, remix, adapt, build upon this work non-commercially, and license their derivative works on different terms, provided the original work is properly cited, appropriate credit is given, any changes made indicated, and the use is non-commercial. See: http://creativecommons.org/licenses/by-nc/4.0/.

\section{ORCID iDs}

Vikas Ostwal http://orcid.org/0000-0002-0067-4748

Sudeep Gupta http://orcid.org/0000-0002-6742-6378

\section{REFERENCES}

1 Marosi C, Köller M. Challenge of cancer in the elderly. ESMO Open 2016;1:e000020.

2 Jemal A, Siegel R, Xu J, et al. Cancer statistics, 2010. CA Cancer J Clin 2010;60:277-300.

3 Patil VM, Chakraborty S, Dessai S, et al. Patterns of care in geriatric cancer patients - an audit from a rural based Hospital cancer registry in Kerala. Indian J Cancer 2015;52:157.

4 Zhao L, Leung L-H, Wang J, et al. Association between Charlson comorbidity index score and outcome in patients with stage IIIB-IV non-small cell lung cancer. BMC Pulm Med 2017;17.

5 Edahyr B, Lind M, Karsera L, et al. PUB068 the applicability of comorbidity indices in predicting chemo toxicity in non-small cell lung cancer. Journal of Thoracic Oncology 2017;12:S1487-8.

6 van Abbema DL, van den Akker M, Janssen-Heijnen ML, et al. Patient- and tumor-related predictors of chemotherapy intolerance in older patients with cancer: a systematic review. J Geriatr Oncol 2019;10:31-41.

7 Hurria A, Togawa K, Mohile SG, et al. Predicting chemotherapy toxicity in older adults with cancer: a prospective multicenter study. JCO 2011;29:3457-65.

8 Hurria A, Mohile S, Gajra A, et al. Validation of a prediction tool for chemotherapy toxicity in older adults with cancer. JCO 2016;34:2366-71.

9 Nie X, Liu D, Li Q, et al. Predicting chemotherapy toxicity in older adults with lung cancer. J Geriatr Oncol 2013;4:334-9.

10 Alibhai SMH, Aziz S, Manokumar T, et al. A comparison of the CArG tool, the VES-13, and oncologist judgment in predicting grade 3+ toxicities in men undergoing chemotherapy for metastatic prostate cancer. J Geriatr Oncol 2017;8:31-6.

11 Yang C-C, Fong Y, Lin L-C, et al. The age-adjusted Charlson comorbidity index is a better predictor of survival in operated lung cancer patients than the Charlson and Elixhauser comorbidity indices. Eur J Cardiothorac Surg 2018;53:235-40.

12 Noronha V, Talreja V, Joshi A, et al. Survey for geriatric assessment in practicing oncologists in India. Cancer Res Stat Treat 2019;2:232.

13 Noronha V, Ramaswamy A, Dhekle R. Initial experience of a geriatric oncology clinic in a tertiary cancer center in India. Cancer Res Stat Treat 2020;3:208.

14 Moth EB, Kiely BE, Stefanic N, et al. Predicting chemotherapy toxicity in older adults: Comparing the predictive value of the CARG Toxicity Score with oncologists' estimates of toxicity based on clinical judgement. J Geriatr Oncol 2019;10:202-9.

15 Extermann M, Boler I, Reich RR, et al. Predicting the risk of chemotherapy toxicity in older patients: the chemotherapy risk assessment scale for High-Age patients (crash) score. Cancer 2012;118:3377-86.

16 Zhang J, Liao X, Feng J, et al. Prospective comparison of the value of crash and CArG toxicity scores in predicting chemotherapy toxicity in geriatric oncology. Oncol Lett 2019;18:4947-55. 